(2) Open Access Full Text Article

\title{
Analysis of the Point Prevalence and Influencing Factors of Acute Stress Disorder in Elderly Patients with Osteoporotic Fractures
}

This article was published in the following Dove Press journal: Neuropsychiatric Disease and Treatment

\section{Qiuke Xiao* \\ Jinwei Ran* \\ Weizhong Lu \\ Ruijie Wan \\ Lujue Dong \\ Zhenyu Dai}

Department of Orthopedics, Chongqing Traditional Chinese Medicine Hospital,

No.4 Clinical Medicine School of

Chengdu University of Traditional

Chinese Medicine, Chongqing 40002I,

People's Republic of China

*These authors contributed equally to this work
Correspondence: Zhenyu Dai Department of Orthopedics, Chongqing Traditional Chinese Medicine Hospital, No.4 Clinical Medicine School of Chengdu University of Traditional Chinese Medicine, Jiangbei District, Chongqing 40002I, People's Republic of China $\mathrm{Tel} / \mathrm{Fax}+862367983672$

Email zhenyudai@cdutcm.edu.cn
Background: Increasing attention has been paid to posttraumatic affective disorders. However, orthopedic surgeons dealing with trauma often ignore the harm of such diseases. Objective: To investigate the point prevalence and influencing factors of acute stress disorder (ASD) in elderly patients with osteoporotic fractures (EPOFs) from the perspective of orthopedic surgeons.

Patients and Methods: A total of 595 cases of EPOFs were treated at our hospital from January 1, 2018, to June 30, 2019. The patients meeting our inclusion criteria were assessed using a structured interview based on the fifth edition of the Diagnostic and Statistical Manual of Mental Disorders (DSM-V) criteria to verify the presence of ASD. After diagnosis, the participants were divided into two groups (those with and without ASD). The sociodemographic characteristics, disease characteristics, and Social Support Rating Scale (SSRS) scores were assessed. The chi-square test was used for univariate analysis, and multivariate analysis was performed using binary logistic regression.

Results: Of the 524 participants, $32(6.1 \%)$ met the criteria for the diagnosis of ASD. The results of the univariate analysis showed that gender, personality, living alone, monthly family income, initial fear, poor prognosis expectation, anxiety/depression, pain, and social support were associated with ASD in EPOFs $(P<0.05)$. The multivariate regression analysis showed that isolation, low monthly family income, introversion, poor prognosis expectation, previous traumatic history, and intense pain were the main influencing factors and risk factors (OR>1) for ASD in EPOFs.

Conclusion: Being female, living alone, introversion, poor family income, intense initial fear, poor prognosis expectation, anxiety/depression, intense pain perception and low social support were significantly related to the occurrence of ASD in EPOFs. To achieve optimal recovery in EPOFs, orthopedic surgeons should meet both the physiological and psychological needs of the patients.

Keywords: acute stress disorder, gender, trauma, fracture, elderly

\section{Introduction}

Trauma is a common experience. The concept of trauma is defined by occurrences in one's life, that are characterized by intensity and one's incapacity to respond suitable to them, the upset they cause, and the lasting psychopathological effects they produce. ${ }^{1}$ It has been estimated that $50 \%$ to $90 \%$ of the US adults experience trauma during their lives. ${ }^{2,3}$ Many victims of trauma recover on their own, but others do not. Acute stress disorder (ASD), an acute trauma response that occurs within 4 weeks following a traumatic event, was introduced as a new diagnosis in 
1994 in the 4th edition of the Diagnostic and Statistical Manual of Mental Disorders (DSM-IV). ${ }^{4}$ In 2013, substantial changes were made to the diagnosis of ASD with the release of the 5 th edition of the DSM (DSM-V). ${ }^{5}$ Patients with ASD respond with intense fear, helplessness, or anguish and may report anxiety, depression, fatigue, headaches, or gastrointestinal or rheumatic symptoms. ${ }^{6}$ Since the introduction of the ASD diagnosis, research on acute posttraumatic stress has been increasing. Previous studies have shown that the prevalence of ASD is $6 \%$ among people who experience an industrial accident, ${ }^{7}$ $13-14 \%$ among people who experience a motor vehicle accident, ${ }^{8,9} 19 \%$ among people who experience a violent assault, ${ }^{10}$ and up to $33 \%$ among people who experience a mass shooting. ${ }^{11}$ However, ASD is not included in largescale psychiatric epidemiology studies because these studies generally focus on chronic diseases. In addition, in terms of the prevalence of ASD, the temporary nature of the disease (within one month) makes it difficult to accurately estimate the epidemiological population ratio. ${ }^{12}$

Osteoporotic fracture is a major and increasing cause of morbidity and loss of independence worldwide, with 5.8 million healthy life years lost to disability annually. ${ }^{13}$ Due to poor bone condition combined with other systemic diseases in elderly patients, treating elderly patients with osteoporotic fractures (EPOFs) has been a challenge for orthopedic surgeons. If EPOFs are suffering from certain mental disorders, such as ASD and posttraumatic stress disorder (PTSD), their poor adherence makes a complicated situation for treatment. Most previous studies on ASD have focused on patients with high-energy injuries caused by road traffic accidents, and few studies have focused on the ASD in elderly patients with fractures caused by low-energy injuries. ${ }^{14-17}$ Compared with the prevalence of ASD in patients with high-energy injuries, that in EPOFs may be relatively low. However, the harm caused by ASD in EPOFs should not be ignored by orthopedic surgeons because it may cause challenges in postoperative nursing care, the failure of early functional exercise, and even the failure of internal fixation due to poor treatment adherence. ${ }^{18,19}$ Clinically, most elderly patients with fractures experience osteoporotic fractures caused by low-energy injuries (injury severity score, ${ }^{20}$ ISS $\leq 16$ ), and severe fractures caused by high-energy injuries (ISS $>16$ ) are rare. Thus, the data of hospitalized EPOFs caused by low-energy injuries between January 1, 2018, and June 30, 2019, in our hospital were collected, and the point prevalence and risk factors for ASD in the EPOFs were investigated from the perspective of orthopedic surgeons in this study.

\section{Patients and Methods}

\section{Patients}

Our consecutive subjects were aged 65 years or older and had been hospitalized for osteoporotic fracture in the orthopedic ward of Chongqing Traditional Chinese Medicine Hospital (CTCMH) between January 1, 2018, and June 30, 2019. The hospitalized EPOFs were assessed using a structured interview based on the DSM-V criteria to verify the presence of ASD. After diagnosis, participants were divided into two groups (those with and without ASD). This study was approved by the Research Ethics Board of CTCMH (No. 2018-ky-DZY), and written informed consent was obtained from all participating patients prior to study commencement.

The inclusion criteria were as follows: patients aged $\geq 65$ years; patients with osteoporotic fractures (ISS $\leq 16$ ); patients with available data from 3 days to 1 month after the injury; and patients with clear consciousness and normal cognitive ability who could fill in the questionnaire or answer the questionnaire independently or with the help of the researchers. The exclusion criteria were as follows: acute trauma resulting in a central nervous system disorder; long-term/intermittent coma or a mental disorder; other severe stress events that occurred during treatment; symptoms of mental illness or a history of mental illness; unclear language expression or a communication barrier; fracture caused by a high-energy traumatic event (ISS score $>16$ ) or a chronic pain history.

\section{Procedures}

Enrolled inpatients were interviewed in person by uniformly trained investigators. The time of psychological evaluation was 3-28 days after injury. The investigation was conducted after dinner, when the patient's emotional state was relatively stable and the environment was relatively quiet. The evaluation factors were as follows:

1. Sociodemographic characteristics: Collected sociodemographic characteristics included gender, personality, living alone status, education, residential area, and monthly family income.

2. Disease characteristics: Disease characteristics were measured using a number of different questions and 
instruments; chronic somatic illnesses, traumatic experience(s), initial fear, prognosis expectation, anxiety/depression, pain and social support were all measured.

Anxiety/depression was assessed by the Hospital Anxiety and Depression Scale (HADS). ${ }^{21}$ Patients with acute trauma are prone to fear, insomnia and other symptoms. The HADS is a self-assessment scale that is used to assess the anxiety and depression of patients in general hospitals, with good reliability and validity. ${ }^{16}$ The HADS includes the anxiety subscale and depression subscale. It has 14 items in total, and each item is divided into 4 grades. The scores of the anxiety and depression subscales are calculated, with a score of 8 considered the cutoff value to determine the presence of anxiety and depression. $^{21}$

The degree of pain was measured on a $100-\mathrm{mm}$ long visual analog scale (VAS). ${ }^{22}$ The VAS has been used for decades and proven to be a valid, reliable and change-sensitive measure of subjective pain. $^{23}$ The patients locate the degree of pain in a straight line according to self-perception (mild pain $\leq 3,3<$ moderate pain $\leq 7$, intense pain $>7$ ). ${ }^{22}$

3. Social Support Rating Scale (SSRS): The SSRS was originally developed for the Chinese population in $1986 .^{24}$ It has been widely used in various studies in different Chinese communities and has been shown to have good validity and reliability. ${ }^{25}$ There are 10 items in the SSRS; 3 items evaluate objective support, 4 items evaluate subjective support and 3 items evaluate support utilization. Objective support reflects objective, visible or practical support received in the past. Subjective support reflects the individual emotional experience of being respected, supported and understood in the community. Support utilization reflects the pattern of behavior that an individual uses when seeking social support. ${ }^{26}$ Higher scores indicate stronger social support (low social support $<20$, $20>$ moderate social support $<30$, satisfactory social support $>30$ ).

\section{Statistical Analysis}

After the data were checked and verified by two persons, Excel software was used to establish the database, and SPSS 22.0 statistical software (IBM Corporation, Armonk, NY, USA) was used for statistical processing. Descriptive statistical methods were used to analyze sociodemographic characteristics; chi-square tests were used for univariate analysis of sociodemographic characteristics, disease characteristics and social support level; and binary logistic regression was used for multivariate analysis.

\section{Results \\ Point Prevalence of ASD in EPOFs}

A total of 595 EPOFs (65 years or older) were admitted to our orthopedic ward from January 1, 2018, to June 30, 2019. A total of 587 (98.7\%) of these patients had mild injuries caused by low-energy events (ISS $<16$ ), 35 patients could not completely fill out the questionnaire, 9 patients were transferred to other departments within 3 days due to concomitant acute cerebral infarction or other special conditions, and 21 patients or family members were not willing to participate in the study. Thus, a total of 524 patients, including 178 male and 346 female patients, met the inclusion criteria. Only 32 (6.1\%) patients met the diagnostic criteria for ASD, and they all met criterion A1 (directly experiencing a traumatic event). In the ASD group, 28 (87.5\%) patients were female, and 4 $(12.5 \%)$ patients were male. A total of $105(20 \%)$ patients fulfilled the criterion of invasive memories, 285 (54.4\%) patients fulfilled the criterion of negative moods, 86 (16.4\%) patients fulfilled the criterion of dissociative symptoms, $136(26 \%)$ patients fulfilled the criterion of avoidance symptoms, and $268(51.1 \%)$ patients fulfilled the criterion of arousal symptoms.

\section{Univariate Analysis \\ Effects of Sociodemographic Characteristics on ASD in EPOFs}

The sociodemographic characteristics are shown in Table 1. The results of the univariate analysis showed that gender, living alone, monthly family income, and personality were related to ASD $(P<0.05)$, with statistically significant differences (Table 2). The risk of ASD in individuals with the characteristics of female gender, living alone, introversion, and low monthly family income was greater than that in individuals with the characteristics of male gender, living with others, extroversion and high monthly family income. Education level and residential area were not related to the occurrence of $\operatorname{ASD}(P>0.05)$.

\section{Effects of Disease Characteristics on ASD in EPOFs} The disease characteristics are shown in Table 3. The results showed that prognosis expectation, anxiety/depression, traumatic history, initial fear and pain were related to 
Table I Sociodemographic Characteristics of All Patients $(\mathrm{n}=524)$

\begin{tabular}{|l|l|l|l|l|}
\hline Sociodemographic Characteristics & \multicolumn{2}{l|}{$\begin{array}{l}\text { ASD } \\
\text { Group }\end{array}$} & \multicolumn{2}{l|}{ Group } \\
\cline { 2 - 5 } & N & $\%$ & N & $\%$ \\
\hline $\begin{array}{l}\text { Gender } \\
\text { Male }\end{array}$ & 4 & 12.5 & 174 & 35.4 \\
Female & 28 & 87.5 & 318 & 64.6 \\
\hline Living alone & & & & \\
Yes & 27 & 84.3 & 53 & 10.8 \\
No & 5 & 15.7 & 439 & 89.2 \\
\hline Personality & & & & \\
Extroversion & 8 & 25.0 & 351 & 71.3 \\
Introversion & 24 & 75.0 & 141 & 28.7 \\
\hline Residential area & & & & \\
Rural & 14 & 43.8 & 215 & 43.7 \\
Urban & 18 & 46.2 & 277 & 56.3 \\
\hline Maximum educational level & & & & \\
Primary school and below & 17 & 53.1 & 248 & 50.4 \\
Junior high school & 12 & 37.5 & 201 & 40.9 \\
Senior high school & 2 & 6.2 & 30 & 6.1 \\
College & 1 & 3.1 & 13 & 2.6 \\
\hline Monthly family income & & & & \\
Good (>9000 RMB) & 3 & 9.4 & 152 & 10.6 \\
Medium (3000-6000 RMB) & 9 & 28.1 & 148 & 30.1 \\
Poor (<3000 RMB) & 20 & 62.5 & 292 & 59.3 \\
\hline
\end{tabular}

the occurrence of ASD in EPOFs $(P<0.05)$, with statistically significant differences. This indicated that patients with poor prognosis expectations, anxiety/depression, intense initial fear, previous traumatic history and intense pain perception were more likely to have an ASD than those with good prognosis expectations, slight pain perception, no anxiety/depression, no traumatic history and initial fear.

\section{Effects of Social Support Level on ASD in EPOFs}

The degree of social support is shown in Table 3. The results showed that social support was associated with the occurrence of ASD in EPOFs $(P<0.05)$, and the difference was statistically significant. This result indicated that the risk of ASD in patients with a low social support level was higher than that in patients with a medium or/and high social support level.

\section{Multivariate Regression Analysis}

The 10 statistically significant factors affecting ASD in the univariate analysis were considered independent variables
(Table 4), and the presence or absence of ASD was included in the binary logistic regression equation as a dependent variable. The results showed that isolation, low monthly family income, introversion, poor prognosis expectation, previous traumatic history, and intense pain were the main influencing factors and risk factors $(\mathrm{OR}>1)$ of ASD (Table 5). No obvious protective factor of ASD was identified $(\mathrm{OR}<1)$.

\section{Discussion}

This study showed that the point prevalence of ASD in EPOFs was related to gender, personality, living alone, initial fear, monthly family income, anxiety/depression, traumatic history, prognosis expectation, social support and pain. Living alone, low monthly family income, introversion, poor prognosis expectation, previous traumatic history, and intense pain were the main influencing factors and risk factors $(\mathrm{OR}>1)$ of ASD. Additionally, our results suggested that the point prevalence of ASD in EPOFs was at the low end of the reported point prevalence but was still in line with previous findings. ${ }^{7,27}$ The relatively low point prevalence of ASD could be attributed to the fact that most of the fractures in elderly patients were caused by low-energy injuries.

The traditional goals for the improvement of treatment are to improve surgical skills and internal fixation materials to maximize recovery of physical function in patients. However, with the reform of the current clinical treatment mode, psychosocial factors play an increasingly significant role in treatment and postoperative rehabilitation. ${ }^{28,29}$ Relevant studies have indicated that early identification of and timely intervention for ASD can greatly reduce its incidence and degree of harm and be very beneficial to the recovery of patients. ${ }^{30-32}$ Therefore, it is important to evaluate the relative risk and protective factors of ASD in EPOFs.

The univariate and logistic regression analyses showed that living alone was a risk factor for ASD in the EPOFs. EPOFs living alone may have an increased risk of ASD because they have greater concern about their future and the stability of their family life and thus tend to have increased anxiety, worry and depression in the face of changes. ${ }^{33}$ This investigation showed that there was no significant correlation between education level and the occurrence of ASD in EPOFs $(P>0.05)$. However, previous studies have suggested that patients with high education levels are more likely to have better medical and disease condition knowledge and are thus less likely prone to anxiety, depression and other negative emotions caused 
Table 2 The Univariate Analysis of Sociodemographic Characteristics

\begin{tabular}{|c|c|c|c|c|c|c|c|c|}
\hline Factors & Category & $\begin{array}{l}\text { ASD } \\
\text { Group }\end{array}$ & $\begin{array}{l}\text { Non-ASD } \\
\text { Group }\end{array}$ & Total & $\chi^{2}$ & $P$ value & $95 \%(\mathrm{Cl})$ & OR \\
\hline Gender & $\begin{array}{l}\text { Female } \\
\text { Male }\end{array}$ & $\begin{array}{l}28 \\
4\end{array}$ & $\begin{array}{l}318 \\
174\end{array}$ & $\begin{array}{l}346 \\
178\end{array}$ & 7.004 & 0.008 & $1.322-11.098$ & 3.830 \\
\hline Living alone & $\begin{array}{l}\text { Yes } \\
\text { No }\end{array}$ & $\begin{array}{l}27 \\
5\end{array}$ & $\begin{array}{l}53 \\
439\end{array}$ & $\begin{array}{l}80 \\
444\end{array}$ & 125.823 & 0.000 & $16.522-121.086$ & 44.728 \\
\hline Monthly family income & $\begin{array}{l}\text { Good } \\
\text { Medium } \\
\text { Poor }\end{array}$ & $\begin{array}{l}3 \\
9 \\
20\end{array}$ & $\begin{array}{l}152 \\
248 \\
92\end{array}$ & $\begin{array}{l}155 \\
257 \\
112\end{array}$ & $\begin{array}{l}{ }^{\mathrm{a}} 0.839 \\
{ }^{\mathrm{b}} 20.937 \\
{ }^{\mathrm{c}} 22.198\end{array}$ & $\begin{array}{l}{ }^{\mathrm{a}} 0.360 \\
{ }^{\mathrm{b}} 0.000 \\
{ }^{\mathrm{c}} 0.000\end{array}$ & $\begin{array}{l}{ }^{\mathrm{a}} 0.490-6.898 \\
\mathrm{~b}_{3} .185-38.092 \\
{ }^{\mathrm{c}} 2.632-13.632\end{array}$ & $\begin{array}{l}{ }^{\mathrm{a}} 1.839 \\
\mathrm{~b} 11.014 \\
{ }^{\mathrm{c}} 5.990\end{array}$ \\
\hline Personality & $\begin{array}{l}\text { Extroversion } \\
\text { Introversion }\end{array}$ & $\begin{array}{l}8 \\
24\end{array}$ & $\begin{array}{l}414 \\
78\end{array}$ & $\begin{array}{l}422 \\
102\end{array}$ & 67.049 & 0.000 & $6.902-36.734$ & 15.923 \\
\hline $\begin{array}{l}\text { Maximum educational } \\
\text { level }\end{array}$ & $\begin{array}{l}\text { Primary school and } \\
\text { below } \\
\text { Junior high school } \\
\text { Senior high school } \\
\text { College }\end{array}$ & $\begin{array}{l}17 \\
12 \\
2 \\
1\end{array}$ & $\begin{array}{l}248 \\
201 \\
30 \\
13\end{array}$ & $\begin{array}{l}265 \\
213 \\
32 \\
14\end{array}$ & $\begin{array}{l}{ }^{d} 0.216 \\
e^{e} 0.001 \\
{ }^{f} 0.012 \\
{ }^{g} 0.020 \\
{ }^{h} 0.055 \\
{ }^{i} 0.013\end{array}$ & $\begin{array}{l}{ }^{d} 0.722 \\
{ }^{e} 0.971 \\
{ }^{f} 0.914 \\
{ }^{g} 0.889 \\
{ }^{h} 0.814 \\
{ }^{i} 0.910\end{array}$ & $\begin{array}{l}{ }^{\mathrm{d}} 0.536-2.460 \\
{ }^{\mathrm{e}} 0.226-4.670 \\
\mathrm{f}^{\mathrm{f}} 0.1 \mathrm{I}-7.222 \\
\mathrm{~g}^{\mathrm{g}} 0.191-4.200 \\
{ }^{\mathrm{h}} 0.094-6.438 \\
{ }^{\mathrm{i}} 0.072-10.423\end{array}$ & $\begin{array}{l}{ }^{d} \mathrm{l} .148 \\
{ }^{\mathrm{e}} \mathrm{l} .028 \\
{ }^{\mathrm{f}} 0.891 \\
{ }^{\mathrm{g}} 0.896 \\
{ }^{\mathrm{h}} 0.776 \\
{ }^{\mathrm{i}} 0.867\end{array}$ \\
\hline Residential area & $\begin{array}{l}\text { Rural } \\
\text { Urban }\end{array}$ & $\begin{array}{l}14 \\
18\end{array}$ & $\begin{array}{l}225 \\
267\end{array}$ & $\begin{array}{l}239 \\
285\end{array}$ & 0.048 & 0.827 & $0.449-1.897$ & 0.923 \\
\hline
\end{tabular}

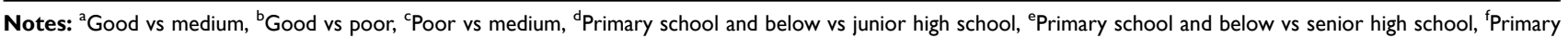
school and below vs college, 'junior high school vs senior high school, hJunior high school vs s college, 'Senior high school vs s college.

Abbreviations: OR, odds ratio; $\mathrm{Cl}$, confidence interval.

Table 3 The Univariate Analysis of Disease Characteristics

\begin{tabular}{|c|c|c|c|c|c|c|c|c|}
\hline Factors & Category & ASD Group & Non-ASD Group & Total & $\chi^{2}$ & $P$ value & $95 \%(\mathrm{Cl})$ & OR \\
\hline \multirow[t]{3}{*}{ Prognosis expectation } & High & I & 278 & 278 & ${ }^{\mathrm{a}} 9.640$ & ${ }^{\mathrm{a}} 0.002$ & ${ }^{\mathrm{a}} 1.615-113.842$ & ${ }^{\mathrm{a}} \mid 3.561$ \\
\hline & Medium & 6 & 123 & 129 & $b_{59.849}$ & b 0.000 & b $10.205-57 \mid .568$ & ${ }^{b} 76.374$ \\
\hline & Low & 25 & 91 & 116 & ${ }^{c} 15.785$ & ${ }^{c} 0.000$ & ${ }^{c} 2.219-14.293$ & ${ }^{c} 5.632$ \\
\hline \multirow[t]{2}{*}{ Traumatic history } & Yes & 15 & 86 & 101 & 16.686 & 0.000 & $2.003-8.663$ & 4.166 \\
\hline & No & 17 & 406 & 423 & & & & \\
\hline \multirow[t]{2}{*}{ Anxiety/depression } & Yes & 25 & 90 & 115 & 62.791 & 0.000 & $6.692-38.028$ & 15.952 \\
\hline & No & 7 & 402 & 409 & & & & \\
\hline \multirow[t]{3}{*}{ Initial fear } & No/slight & 5 & 291 & 296 & ${ }^{d} 4.733$ & ${ }^{d} 0.030$ & ${ }^{\mathrm{d}} 0.097-0.942$ & ${ }^{d} 0.303$ \\
\hline & Moderate & 8 & $|4|$ & 149 & $e^{e} 52.051$ & ${ }^{e} 0.000$ & ${ }^{e} 0.019-0.151$ & ${ }^{\mathrm{e}} 0.054$ \\
\hline & Intense & 19 & 60 & 79 & ${ }^{f} \mid 7.259$ & f 0.000 & fo. $074-0.432$ & fo. 179 \\
\hline \multirow[t]{3}{*}{ Pain } & No/slight & 6 & 109 & 115 & ${ }^{8} 0.691$ & ${ }^{g} 0.406$ & ${ }^{g} 0.549-4.359$ & $\mathrm{~g} / .547$ \\
\hline & Moderate & 10 & 281 & 291 & $\mathrm{~h}_{4.740}$ & ho.029 & ${ }^{h} 0.132-0.932$ & ${ }^{h} 0.351$ \\
\hline & Intense & 16 & 102 & 118 & 'I 4.452 & '0.000 & '0.100-0.516 & '0.227 \\
\hline \multirow[t]{3}{*}{ Social support } & Low & 15 & 125 & 140 & i 4.108 & j0.043 & ${ }^{\mathrm{j}} 1.009-5.318$ & $i_{2.316}$ \\
\hline & Medium & 10 & 193 & 203 & $\mathrm{k}_{5} .797$ & $\mathrm{k}^{\mathrm{N}} 0.016$ & k $1.181-7.531$ & $k_{2} .983$ \\
\hline & High & 7 & 174 & 181 & '0.253 & '0.615 & '0.480-3.457 & '।.288 \\
\hline
\end{tabular}

Notes: ${ }^{\mathrm{a}} \mathrm{High}$ vs medium, ${ }^{\mathrm{b}} \mathrm{High}$ vs low, ${ }^{\mathrm{c}} \mathrm{High}$ vs medium, ${ }^{\mathrm{d}} \mathrm{No} /$ slight vs moderate, ${ }^{\mathrm{e}} \mathrm{No} / \mathrm{slight}$ vs intense, ${ }^{\mathrm{f}}$ Moderate vs intense, ${ }^{\mathrm{g}} \mathrm{No} / \mathrm{slight}$ vs moderate, ${ }^{\mathrm{h}} \mathrm{No} / \mathrm{slight}$ vs intense, 'Moderate vs intense, 'Low vs medium, 'Low vs high, 'High vs medium.

Abbreviations: OR, odds ratio; $\mathrm{Cl}$, confidence interval.

by errors and negative news than those with low education levels; accordingly, they can actively respond to and positively face the difficulties in front of them. ${ }^{34}$ We think that the reason why the results of this study were different from those of previous studies may be related to differences in the cultural levels of patients. The logistic regression 
Table 4 Independent Variable Assignment Table

\begin{tabular}{|l|l|l|}
\hline & Variable & Assignment Method \\
\hline Gender & $\mathrm{X} 1$ & 2=female, I=male \\
Living along & $\mathrm{X} 2$ & 2=yes, I= no \\
Monthly family income & $\mathrm{X} 3$ & 3=poor, 2=medium, I=good \\
Personality & $\mathrm{X} 4$ & 2=introversion, I=extroversion \\
Prognosis expectation & $\mathrm{X} 5$ & 3=poor, 2=medium, I=good \\
Traumatic history & $\mathrm{X6}$ & 2=yes, I=no \\
Anxiety/depression & $\mathrm{X} 7$ & 2=yes, I=no \\
Initial fear & $\mathrm{X8}$ & 3=intense, 2=medium, I=mild/no \\
Pain & $\mathrm{X} 9$ & 3=intense, 2=medium, I=mild/no \\
Social support & $\mathrm{X} 10$ & 3=low, 2=medium, I=high \\
Whether or not ASD & $\mathrm{Y}$ & 2=yes, I=no \\
\hline
\end{tabular}

analysis showed that introversion was a risk factor for the development of ASD in EPOFs, and introverts are more likely to have ASD than extroverts. Other similar studies have also indicated that patients with introverted tendencies experience challenges communicating with the outside world; thus, they receive less attention and support from others and are more prone to posttraumatic mental disorders after stress stimulation than those with extroverted tendencies. ${ }^{35}$ Our results also showed that low monthly family income was a risk factor for ASD in EPOFs. After a traumatic accident, people with low incomes may experience increased psychological and functional difficulties due to a lack of mediating variables such as occupational, social and financial resources. ${ }^{36}$ The conservation of resources theory suggests that low income may be a chronic stressor, increasing psychological distress as a result of limited access to resources and resource accumulation. $^{37}$

The results of the univariate analysis showed that gender was related to ASD, as the point prevalence of ASD in females was higher than that in males $(P<0.05)$. This is in line with previous research results. ${ }^{18,38}$ Bryant and Harvey reported that $23 \%$ of women experienced ASD after motor vehicle accidents, while only $8 \%$ of men experienced ASD. ${ }^{39}$ Previous studies have suggested that gender differences in mental response during a disaster may be related to differences in the encoding of memories of disasters and differences in cognitive schemas between the sexes. ${ }^{40}$ This suggests that it is necessary to provide more psychological counseling and care to elderly female patients with traumatic fractures.

The analysis of disease characteristics showed that previous traumatic experience(s), poor prognosis expectation and intense pain perception were risk factors $(\mathrm{OR}>1)$ for the development of ASD in EPOFs. Previous reports have shown that patients with previous traumatic experiences are increasingly likely to develop ASD when they are faced with trauma again. ${ }^{41,42}$ This may be due to the recall of events, scenarios or other clues related to the traumatic events or similar events, and adverse scenario stimuli lead to fear, which results in strong psychological pain and physiological reactions. Anxiety is commonly comorbid with depression disorders and $\mathrm{ASD}^{43-45}$ Anxiety/depression is a mood disorder characterized by an anxious/depressed mood or loss of interest or pleasure, with neurovegetative and cognitive symptoms. ${ }^{46}$ Posttraumatic anxiety/depression often occurs when patients realize that their health has been greatly damaged and they are facing a risk of dysfunction and disability; this generally occurs after "emotional shock" in the early stage of trauma. Our results showed that anxiety/depression was related to ASD, which is consistent with other studies that have noted that patients with a predisposition to anxiety are prone to developing clinically significant stress responses in relation to life stressors. ${ }^{47-49}$

Table 5 The Binary Logistic Regression Analysis of ASD

\begin{tabular}{|l|l|l|l|l|l|l|}
\hline Factor & B & SE & Wald & P & 95\%(CI) \\
\hline Gender & 0.269 & 1.071 & 0.063 & 0.802 & $0.160-10.665$ & OR \\
Living alone & 4.303 & 1.286 & 11.190 & 0.001 & $5.941-920.007$ & 1.308 \\
Monthly family income & 2.050 & 0.800 & 6.575 & 0.010 & $1.621-37.246$ & 73.930 \\
Personality & 4.190 & 1.330 & 9.928 & 0.002 & $4.873-894.405$ & 7.771 \\
Prognosis expectation & 2.005 & 0.754 & 7.083 & 0.008 & $1.696-32.535$ & 66.015 \\
Traumatic history & 2.983 & 1.286 & 5.384 & 0.020 & $1.589-245.522$ & 7.429 \\
Anxiety/depression & 1.738 & 1.058 & 2.698 & 0.101 & $0.715-45.246$ & 19.753 \\
Initial fear & 1.585 & 0.825 & 3.696 & 0.055 & $0.970-24.571$ & 5.686 \\
Pain & 1.454 & 0.823 & 3.117 & 0.077 & $0.852-21.499$ & 4.881 \\
Social support & 1.167 & 0.674 & 2.998 & 0.083 & $0.857-12.041$ & 4.280 \\
\hline
\end{tabular}

Abbreviations: OR, odds ratio; $\mathrm{Cl}$, confidence interval. 
Pain is a common clinical manifestation of many diseases, and a concern of both doctors and patients. It is an experience that we associate with actual or potential tissue damage. Pain is unquestionably a sensation in a part or parts of the body, but it is also always unpleasant and therefore also an emotional experience. ${ }^{50}$ Consistent with previous literature, ${ }^{51,52}$ our results also showed that pain is a risk factor for ASD. In addition, many studies have verified the relationship between pain and PTSD. In patients with whiplash injury, pain was associated with acute posttraumatic psychological responses. ${ }^{53}$ In victims of traffic accidents, pain was found to be related to PTSD morbidity. ${ }^{54}$ In another study of victims of motor vehicle accidents, a reduction in pain severity was a significant predictor of a reduction in PTSD symptoms. ${ }^{55}$ Fuglsang et al found that pain can be a powerful trigger of reexperiencing symptoms, thus repeatedly reminding patients of the trauma they have experienced. ${ }^{56}$ These findings suggest that effective relief of posttraumatic pain may help reduce the incidence rate of ASD.

Social support is generally defined as the belief that one is cared for, loved, respected, and available for help and serves as a protective factor for emotional disorders. ${ }^{57}$ Social support comprises multiple dimensions, including the quantity and quality of support and perceived social support, and may be derived from multiple sources, including family, friends, colleagues and the community. ${ }^{58}$ Previous studies have demonstrated that satisfactory social support can reduce the occurrence of psychological stress disorder to a certain extent, improve the ability of injured patients to handle traumatic events, and provide long-term protection in individuals through mediators, ${ }^{59}$ while poor social support is associated with depression, ${ }^{60}$ suicide, ${ }^{61,62}$ anxiety disorders, schizophrenia, and bipolar disorder. ${ }^{63}$ Our results showed that low social support is a risk factor for ASD. Similar to our research results, Sattler et al found that low social support was related to the occurrence of ASD, and patients with low social support were more likely to develop ASD, promoting the development of ASD symptoms. ${ }^{64}$ Therefore, if family members, relatives, friends, medical staff and social groups of all levels provide broad emotional, economic and psychological support to traumatized individuals through a variety of channels, the occurrence of posttraumatic ASD may be reduced.

PTSD is a common mental disorder manifesting through symptoms of reexperiencing, hyper-arousal and avoidance following a traumatic event and is associated with substantial physical and psychiatric co-morbidities, including substance abuse and suicide. ${ }^{65}$ The DSM-V states that symptoms must be present for one month following the traumatic event for PTSD to be diagnosed. While the ASD has similar symptoms to PTSD, it is diagnosed 3 days to 1 -month post-trauma. The previous literature has indicated that it is probable that most risk factors for PTSD also apply to ASD, and ASD was introduced partly to predict subsequent PTSD, but longitudinal studies indicate that ASD is not an accurate predictor of PTSD. ${ }^{17,66}$ However, a multisite study found that subsyndromal ASD criteria that did not require dissociation to be present had greater sensitivity for predicting the subsequent development of PTSD than the DSM-IV ASD criteria. ${ }^{67}$ Therefore, long-term follow-up to observe the relationship between subsyndromal ASD and PTSD should be conducted in our next study. In addition, another review has questioned the validity and limitations of ASD as a diagnosis; ${ }^{68}$ the definition of dissociation (a central element of ASD diagnosis) is still unclear, what peritraumatic is dissociation and its association with ASD are still in doubt, and there is difficulty in establishing what qualifies as a clinically significant level of distress or impairment (Criterion F for ASD). However, although the complexity of the posttraumatic stress process limits the diagnosis of ASD and the prediction of subsequent PTSD, the use of existing DSM diagnostic criteria still plays a positive role in the early prevention and treatment of posttraumatic emotional disorders. Certainly, more longitudinal studies should be conducted to gain a better understanding of the long-term reaction to trauma, to assess somatic symptoms, and to investigate the effect of stress on morbidity or on worsening of illness as a reaction to exposure to traumatic events.

Several limitations in this study are evident. First, the relatively small sample size may have weakened the statistical power. Second, stressful life event data were collected retrospectively, and recall bias may have affected the results. Third, no professional psychiatrists participated in this study; thus, whether the questions answered by the super elderly patients ( $>85$ years) were completely accurate is questionable. Fourth, the DSM adopts a consensual and syndromal check-list approach that is largely theoretical. ${ }^{69}$ Therefore, some subjective errors may occur in the collection of research data, including patients and investigators. However, the original purpose of this study was to observe patients' emotional disorders from the perspective of surgeons; thus, we believe that the results of this study still have relatively good clinical significance for surgeons.

In conclusion, surgeons operating on elderly fracture patients should provide help and support to patients 
considering the above possible risk factors for ASD, encourage patients' families and friends to provide emotional and material support, and guide patients to utilize their social support system effectively to avoid the occurrence and deterioration of ASD. As only orthopedic surgeons were involved in this study, we hope that future research jointly includes surgical staff and psychiatrists so that the results of the research are more clinically significant.

\section{Acknowledgments}

This work was supported by the plan of high-level medical reserved personnel training project of Chongqing (No. 2017HBRC012).

\section{Disclosure}

The authors report no conflicts of interest in this work.

\section{References}

1. Santana MRM, Zatti C, Spader ML, et al. Acute stress disorder and defense mechanisms: a study of physical trauma patients admitted to an emergency hospital. Trends Psychiatry Psychother. 2017;39 (4):247-256. doi:10.1590/2237-6089-2016-0071

2. Kessler RC, Sonnega A, Bromet E, Hughes M, Nelson CB. Posttraumatic stress disorder in the National Comorbidity Survey. Arch Gen Psychiatry. 1995;52(12):1048-1060. doi:10.1001/ archpsyc.1995.03950240066012

3. Breslau N, Kessler RC, Chilcoat HD, Schultz LR, Davis GC, Andreski P. Trauma and posttraumatic stress disorder in the community: the 1996 Detroit area survey of trauma. Arch Gen Psychiatry. 1998;55(7):626-632. doi:10.1001/archpsyc.55.7.626

4. Battle DE; American Psychiatric Association. Diagnostic and Statistical Manual of Mental Disorders. 4th ed. Washington DC; 1994

5. Battle DE; American Psychiatric Association. Diagnostic and Statistical Manual of Mental Disorders. Fifth ed (DSM-5). Arlington: American Psychiatric Publishing; 2013

6. Forbes D, Creamer M, Phelps A, et al. Australian guidelines for the treatment of adults with acute stress disorder and post-traumatic stress disorder. Aust N Z J Psychiatry. 2007;41(8):637-648. doi:10. 1080/00048670701449161

7. Creamer M, Manning C. Acute stress disorder following an industrial accident. Aust Psychol. 1998;33:125-129. doi:10.1080/00050069808 257393

8. Bryant RA, Harvey AG. Relationship between acute stress disorder and posttraumatic stress disorder following mild traumatic brain injury. Am J Psychiatry. 1998;155:625-629. doi:10.1176/ajp.155.5. 625

9. Harvey AG, Bryant RA. The Relationship between acute stress disorder and posttraumatic stress disorder: a prospective evaluation of motor vehicle accident survivors. J Consult Clin Psychol. 1998; 66:507-512.

10. Brewin CR, Andrews B, Rose S, Kirk M. Acute stress disorder and posttraumatic stress disorder in victims of violent crime. $\mathrm{Am}$ J Psychiatry. 1999;156:360-366.

11. Classen C, Koopman C, Hales R, Spiegel D. Acute stress disorder as a predictor of posttraumatic stress symptoms. Am J Psychiatry. 1998;155:620-624. doi:10.1176/ajp.155.5.620
12. Bryant RA. Acute stress disorder. Curr Opin Psychol. 2017;14:127-131. doi:10.1016/j.copsyc.2017.01.005

13. Alarkawi D, Bliuc D, Tran T, et al. Impact of osteoporotic fracture type and subsequent fracture on mortality: the tromsø study. Osteoporos Int. 2020;1(1):119-130. doi:10.1007/s00198-019-05 174-5

14. Dai W, Liu A, Kaminga AC, et al. Prevalence of acute stress disorder among road traffic accident survivors: a meta-analysis. $B M C$ Psychiatry. 2018;18(1):188. doi:10.1186/s12888-018-1769-9

15. Sijbrandij M, Kleiboer A, Bisson JI, Barbui C, Cuijpers P. Pharmacological prevention of post-traumatic stress disorder and acute stress disorder: a systematic review and meta-analysis. Lancet Psychiatry. 2015;2(5):413-421. doi:10.1016/S2215-0366(14)00121-7

16. Astill Wright L, Sijbrandij M, Sinnerton R, et al. Pharmacological prevention and early treatment of post-traumatic stress disorder and acute stress disorder: a systematic review and meta-analysis. Transl Psychiatry. 2019;9(1):334. doi:10.1038/s41398-019-0673-5

17. Bryant RA. The current evidence for acute stress disorder. Curr Psychiatry Rep. 2018;20(12):111.

18. Cohen M. Acute stress disorder in older, middle-aged and younger adults in reaction to the second Lebanon war. Int $J$ Geriatr Psychiatry. 2008;23(1):34-40. doi:10.1002/gps.1832

19. Cahill SP, Pontoski K. Post-traumatic stress disorder and acute stress disorder I: their nature and assessment considerations. Psychiatry (Edgmont). 2005;4:14-25.

20. Baker SP, O’Neill B, Haddon W Jr, et al. The injury severity score: a method for describing patients with multiple injuries and evaluating emergency care. J Trauma. 1974;14(3):187-196. doi:10.1097/00005 373-197403000-00001

21. Zigmond AS, Snaith RP. The hospital anxiety and depression scale. Acta Psychiatr Scand. 1983;67(6):361-370. doi:10.1111/j.16000447.1983.tb09716.x

22. Huskisson EC. Measurement of pain. Lancet. 1974;2(7889): 1127-1131. doi:10.1016/S0140-6736(74)90884-8

23. Bijur PE, Silver W, Gallagher EJ. Reliability of the visual analog scale for measurement of acute pain. Acad Emerg Med. 2001;8 (12):1153-1157. doi:10.1111/j.1553-2712.2001.tb01132.x

24. Yu Y, Yang JP, Shiu CS, et al. Psychometric testing of the Chinese version of the medical outcomes study social support survey among people living with HIV/AIDS in China. Appl Nurs Res. 2015;28 (4):328-333. doi:10.1016/j.apnr.2015.03.006

25. Shao R, He P, Ling B, et al. Prevalence of depression and anxiety and correlations between depression, anxiety, family functioning, social support and coping styles among Chinese medical students. $B M C$ Psychol. 2020;8(1):38.

26. Ahmad S, Feder A, Lee EJ, et al. Earthquake impact in a remote South Asian population: psychosocial factors and posttraumatic symptoms. J Trauma Stress. 2010;23(3):408-412. doi:10.1002/jts.20 535

27. Grieger TA, Staab JP, Cardena E, et al. Acute stress disorder and subsequent post-traumatic stress disorder in a group of exposed disaster workers. Depress Anxiety. 2000;11(4):183-184. doi:10.10 02/1520-6394(2000)11:4<183::AID-DA7>3.0.CO;2-M

28. Gong L, Chen H. Descriptive analysis of the cost-effectiveness of depressed patients undergoing total knee arthroplasty: an economic decision analysis. J Orthop Sci. 2014;19(5):820-826. doi:10.1007/ s00776-014-0599-y

29. Fortinsky RH, Bohannon RW, Litt MD, et al. Rehabilitation therapy self-efficacy and functional recovery after hip fracture. Int $J$ Rehabil Res. 2002;25(3):241-246. doi:10.1097/00004356-200209000-00011

30. Humar A, Morris M, Blumberg E, et al. Nucleic acid testing(NAT) of organ donors: is the "best" test the right test? A consensus conference report. Am Jransplant. 2010;10(4):889-899.

31. Roberts NP, Kitchiner NJ, Kenardy J, et al. Early psychological interventions to treat acute traumatic stress symptoms. Cochrane Database Syst Rev. 2010;3:CD007944. 
32. Peris A, Bonizzoli M, Iozzelli D, et al. Early intra-intensive care unit psychological intervention promotes recovery from post traumatic stress disorders, anxiety and depression symptoms in critically ill patients. Crit Care. 2011;15(1):R41. doi:10.1186/cc10003

33. Chen Y, Hicks A, While AE. Loneliness and social support of older people living alone in a county of Shanghai, China. Health Soc Care Community. 2014;22(4):429-438. doi:10.1111/hsc.12099

34. Zhao D, Hu C, Chen J, et al. Risk factors of geriatric depression in rural China based on a generalized estimating equation. Int Psychogeriatr. 2018;30(10):1489-1497. doi:10.1017/S1041610 218000030

35. Holeva V, Tarrier N. Personality and peritraumatic dissociation in the prediction of PTSD in victims of road traffic accidents. J Psychosom Res. 2001;51(5):687-692. doi:10.1016/S0022-3999(01)00256-2

36. Dorahy MJ, Rowlands A, Renouf C, et al. Impact of average household income and damage exposure on post-earthquake distress and functioning: a community study following the February 2011 Christchurch earthquake. $\mathrm{Br} J$ Psychol. 2015;106(3):526-543. doi:10.1111/bjop.12097

37. Ennis NE, Hobfoll SE, Schröder KE. Money doesn't talk, it swears: how economic stress and resistance resources impact inner-city women's depressive mood. Am J Community Psychol. 2000;28 (2):149-173. doi:10.1023/A:1005183100610

38. Tolin DF, Foa EB. Sex differences in trauma and posttraumatic stress disorder: a quantitative review of 25 years of research. Psychol Bull. 2006;132:959-992. doi:10.1037/0033-2909.132.6.959

39. Bryant RA, Harvey AG. Gender differences in the relationship between acute stress disorder and posttraumatic stress disorder following motor vehicle accidents. Aust $N Z$ J Psychiatry. 2003;37:226-229. doi:10.1046/j.1440-1614.2003.01130.x

40. Simmons CA, Granvold DK. A cognitive model to explain gender differences in rate of PTSD diagnosis. Brief Treat Crisis Interv. 2005;5:290-299. doi:10.1093/brief-treatment/mhi021

41. Fuglsang AK, Moergeli H, Schnyder U. Does acute stress disorder predict posttraumatic stress disorder in traffic accident victims? A nalysis of a self-report inventory. Nord J Psychiatry. 2004;58 (3):223-229. doi:10.1080/08039480410006278

42. Meiser-Stedman R, Dalgleish T, Smith P, et al. Diagnostic, demographic, memory quality and cognitive variables associated with acute stress disorder in children and adolescents. $J$ Abnorm Psychol. 2007;116(1):65-79. doi:10.1037/0021-843X.116.1.65

43. Blanchard EB, Buckley TC, Hickling EJ, et al. Posttraumatic stress disorder and comorbid major depression: is the correlation an illusion? J Anxiety Disord. 1998;12(1):21-37. doi:10.1016/S08876185(97)00047-9

44. Shalev AY, Freedman S, Peri T, et al. Prospective study of posttraumatic stress disorder and depression following trauma. Am J Psychiatry. 1998;155(5):630-637. doi:10.1176/ajp.155.5.630

45. Harvey AG, Bryant RA. Predictors of acute stress disorder following mild traumatic brain injury. Brain Inj. 1998;12(2):147-154. doi:10. 1080/026990598122773

46. Koenig HG, Cohen HJ, Blazer DG, et al. Religious coping and cognitive symptoms of depression in elderly medical patients. Psychosomatics. 1995;36(4):369-375. doi:10.1016/S0033-3182(95) 71646-2

47. Barton KA, Blanchard EB, Hickling EJ. Antecedents and consequences of acute stress disorder among motor vehicle accident victims. Behav Res Ther. 1996;34(10):805-813. doi:10.1016/00057967(96)00027-7

48. Franklin CL, Zimmerman M. Posttraumatic stress disorder and major depressive disorder: investigating the role of overlapping symptoms in diagnostic comorbidity. J Nerv Ment Dis. 2001;189(8):548-551. doi:10.1097/00005053-200108000-00008

49. Suliman S, Troeman Z, Stein DJ, et al. Predictors of acute stress disorder severity. J Affect Disord. 2013;149(1-3):277-281. doi:10.10 16/j.jad.2013.01.041
50. Merskey H, Bogduk N. Classification of Chronic Pain. 2nd, IASP Task Force on Taxonomy. Seattle: IASP Press; 1994.

51. Kassam-Adams N, Fleisher CL, Winston FK. Acute stress disorder and posttraumatic stress disorder in parents of injured children. J Trauma Stress. 2009;22(4):294-302. doi:10.1002/jts.20 424

52. Saxe GN, Miller A, Bartholomew D, et al. Incidence of and risk factors for acute stress disorder in children with injuries. J Trauma. 2005;59(4):946-953. doi:10.1097/01.ta.0000187659.373 85.16

53. Drottning M, Staff PH, Levin L, Malt UF. Acute emotional response to common whiplash predicts subsequent pain complaints: a prospective study of 107 subjects sustaining whiplash injury. Nord J Psychiat. 1995;49:293-300. doi:10.3109/08039489509011 919

54. Kuch K, Cox BJ, Evans RJ. Posttraumatic stress disorder and motor vehicle accidents: a multidisciplinary overview. Can J Psychiatry. 1996;41(7):429-434

55. Fedoroff IC, Taylor S, Asmundson GJG, Koch WJ. Cognitive factors in traumatic stress reactions: predicting PTSD symptoms from anxiety sensitivity and beliefs about harmful events. Behav Cogn Psychother. 2000;28:5-15. doi:10.1017/S1352465800000023

56. Fuglsang AK, Moergeli H, Hepp-Beg S, Schnyder U. Who develops acute stress disorder after accidental injuries? Psychother Psychosom. 2002;71(4):214-222. doi:10.1159/000063647

57. Eker D, Arkar H. Perceived social support: psychometric properties of the MSPSS in normal and pathological groups in a developing country. Soc Psychiatry Psychiatr Epidemiol. 1995;30(3):121-126. doi:10.1007/BF00802040

58. Norris FH, Kaniasty K. Received and perceived social support in times of stress: a test of the social support deterioration deterrence model. J Pers Soc Psychol. 1996;71(3):498-511. doi:10.1037/00223514.71.3.498

59. Adriaenssens J, de Gucht V, Maes S. The impact of traumatic events on emergency room nurses: findings from a questionnaire survey. Int J Nurs Stud. 2012;49(11):1411-1422. doi:10.1016/j.ijnurstu.20 12.07 .003

60. Bell CM, Ridley JA, Overholser JC, et al. The role of perceived burden and social support in suicide and depression. Suicide Life Threat Behav. 2018;48(1):87-94. doi:10.1111/sltb.12327

61. Kleiman EM, Liu RT. Social support as protective factor in suicide: findings from two nationally representative samples. J Affect Disord. 2013;150(2):540-545. doi:10.1016/j.jad.2013.01.033

62. Marver JE, Galfalvy HC, Burke AK, et al. Friendship, depression, and suicide attempts in adults: exploratory analysis of longitudinal follow-up study. Suicide Life Threat Behav. 2017;47(6):660-671. doi:10.1111/sltb.12329

63. Wang Y, Sareen J, Afifi TO, et al. A population-based longitudinal study of recent stressful life events as risk factors for suicidal behavior in major depressive disorder. Arch Suicide Res. 2015;19 (2):202-217. doi:10.1080/13811118.2014.957448

64. Sattler DN, de Alvarado AM, de Castro NB, et al. El Salvador earthquakes: relationships among acute, stress disorder symptoms, depression, traumatic event exposure, and resource loss. J Trauma Stress. 2006;19(6):879-893. doi:10.1002/jts.20174

65. Bjelland I, Dahl AA, Haug TT, Neckelmann D. The validity of the hospital anxiety and depression scale. An updated literature review. J Psychosom Res. 2002;52(2):69-77. doi:10.1016/S0022-3999(01) 00296-3

66. Harvey AG, Bryant RA. Predictors of acute stress following motor vehicle accidents. J Trauma Stress. 1999;12(3):519-525. doi:10.10 23/A:1024723205259

67. Bryant RA, Creamer M, O’Donnell ML, Silove D, McFarlane AC. A multisite study of the capacity of acute stress disorder diagnosis to predict posttraumatic stress disorder. J Clin Psychiatry. 2008;69 (6):923-929. doi:10.4088/JCP.v69n0606 
68. Cardeña E, Carlson E. Acute stress disorder revisited. Annu Rev Clin Psychol. 2011;7:245-267. doi:10.1146/annurev-clinpsy-03221 $0-104502$
69. Ng QX, Lim DY, Chee KT. Reimagining the spectrum of affective disorders [published online ahead of print, 2020 Jun 18]. Bipolar Disord. 2020. doi:10.1111/bdi.12960

\section{Publish your work in this journal}

Neuropsychiatric Disease and Treatment is an international, peerreviewed journal of clinical therapeutics and pharmacology focusing on concise rapid reporting of clinical or pre-clinical studies on a range of neuropsychiatric and neurological disorders. This journal is indexed on PubMed Central, the 'PsycINFO' database and CAS, and is the official journal of The International Neuropsychiatric Association (INA). The manuscript management system is completely online and includes a very quick and fair peer-review system, which is all easy to use. Visit http://www.dovepress.com/testimonials.php to read real quotes from published authors. 